\title{
IMPACTO DE IMPLEMENTACIÓN DE LAS NORMAS INTERNACIONALES DE CONTABILIDAD PARA EL SECTOR PÚBLICO EN EL MUNICIPIO DE TEBAIDA DEL DEPARTAMENTO DEL QUINDIO
}

\section{IMP ACT OF IMPLEMENTATION OF INTERNATIONAL ACCOUNTING STANDARDS FOR THE PUBLIC SECTOR IN THE MUNICIP ALITY OF TEBAIDA OF THE QUINDIO DEPARTMENT}

\author{
Olga Inés Ceballos Rincón ${ }^{1}$ \\ Luz Amparo Mejía Castellanos ${ }^{2}$ \\ Deicy Arango Medina ${ }^{3}$
}

Cómo citar esté artículo: Ceballos Rincón, O. I., Mejía Castellanos, L. A. \& Arango Medina, D. (2020). Impacto de implementación de las normas internacionales de contabilidad para el sector público en el municipio de Tebaida del departamento del Quindío. Revista Innova ITFIP, 7(1), 89-109

Recibido: Mayo de 2020. Aprobado: Noviembre de 2020

\section{Resumen}

Este articulo busca, describir el impacto de las normas internacionales de Contabilidad para el sector público en el municipio de Tebaida del departamento del Quindío, generado por la implementación de la Resolución 533 del 2015, en el estado de situación financiera de apertura. Es una investigación descriptiva e inductiva, por cuanto identifica características particulares de las entidades en referencia, como lo es su naturaleza jurídica, económica y social; además, el estudio fue documental teniendo en cuenta que el entorno en el que se desarrolló es público y por lo tanto fue necesario el uso de información oficial para la recolección y análisis de datos; por otra parte, se hizo un análisis transversal, debido a que la recolección de datos se dio en un momento específico y responde a un problema particular, que para el caso es el impacto de la implementación de esta Resolución en el estado de situación financiera de apertura, en las entidades de gobie rno. Como consecuencia de este estudio, se evidenció que las políticas contables fueron adecuadas para cada uno de los elementos del estado de situación financiera a excepción de algunas partidas en las cuales no se especificó el método de medición, el tiempo de vida útil y los criterios para el reconocimiento.

\footnotetext{
${ }^{1}$ Docente investigador de la Universidad del Quindío. Armenia, Colombia. ORCID: https://orcid.org/ 0000-00024082-6451. Correo electrónico: oiceballos@uniquindio.edu.co

${ }^{2}$ Docente investigador de la Universidad del Quindío. Armenia, Colombia. ORCID: https://orcid.org/ 0000-00025701-8987. Correo electrónico: luza@uniquindio.edu.co

${ }^{3}$ Docente investigador de la Universidad del Quindío. Armenia, Quindío. ORCID: https://orcid.org/0000-0002-76035563. Correo electrónico: darango@uniquindio.edu.co
} 
REVISTA INNOVA ITFIP, 7 (1). 89-109 DIC. 2020

Una vez identificada la estructura de las políticas contables se describió el impacto en el estado de situación financiera de apertura, generado por la implementación de la Resolución, aplicando herramientas de análisis e interpretación de los estados financieros, como resultado de este proceso, se obtuvo cifras porcentuales de participación de cada una de las cuentas con sus respectivas variaciones, las más significativas, fueron el Activo en cuanto a Efectivo y Equivalente al Efectivo, Inversiones e Instrumentos Derivados y el Pasivo en las Provisiones, las cuales hicieron parte del saneamiento contable para la transición al nuevo marco normativo, el cual tuvo un impacto significativo en el patrimonio de la entidad.

Para concluir, la administración municipal deberá cumplir efectivamente con todos los requisitos expuestos por la Contaduría General de la Nación para ejecutar eficientemente las Normas Internacionales de Contabilidad del Sector Público bajo el nuevo marco normativo, además de seguir estableciendo todas aquellas acciones y estrategias necesarias para que exista un adecuado, oportuno flujo de información y documentación hacia el área contable, con el propósito de garantizar el reconocimiento y revelación de todos los hechos económicos de la misma.

\title{
Palabras Clave
}

Políticas contables, Normas Internacionales de Contabilidad para el Sector Público, Contaduría General de la Nación, Resolución 533.

\begin{abstract}
This article seeks to describe the impact of international accounting standards for the public sector on the municipality of Tebaida of the Quindío department, generated by the implementation of Resolution 533 of 2015, on the state of open financial position. It is a descriptive and inductive investigation, in that it identifies particular characteristics of the entities in reference, such as their legal, economic and social nature; In addition, the study was documentary considering that the environment in which it was developed is public and therefore it was necessary to use official information for data collection and analysis; on the other hand, a cross-cutting analysis was done, because the data collection was given at a specific time and responds to a particular problem, which for that matter is the impact of the implementation of this Resolution on the state of financial situation of openness, on government entities. As a result of this study, it was shown that accounting policies were appropriate for each element of the financial statement except for some items in which the measurement method, useful time, and criteria for recognition were not specified.
\end{abstract}

Once the structure of accounting policies was identified, the impact on the opening financial statement, generated by the implementation of the Resolution, was described by applying tools for 
REVISTA INNOVA ITFIP, 7 (1). 89-109 DIC. 2020

the analysis and interpretation of financial statements, as a result of this process, percentage figures were obtained from each of the accounts with their respective variations, the most significant, were the Asset in terms of Cash and Cash Equivalent, Investments and Derivative Instruments and the Liabilities in the Provisions, which were part of the accounting consolidation for the transition to the new regulatory framework, which had a significant impact on the assets of the entity.

In conclusion, the municipal administration must effectively comply with all the requirements set out by the Attorney General's Office to efficiently implement the International Public Sector Accounting Standards under the new regulatory framework, in addition to continuing to establish all those actions and strategies necessary for an adequate, timely flow of information and documentation to the accounting area, with the purpose of ensuring the recognition and disclosure of all economic facts thereof.

\section{Keywords}

Accounting policies, International Accounting Standards for the Public Sector, General Accounting Office, Resolution 533.

\section{Introducción}

Las entidades y empresas del estado colombiano, tienen la obligación de dar cumplimiento a la implementación de las Normas Internacionales de Contabilidad para el Sector Público, de acuerdo con los lineamientos establecidos por la Contaduría General de la Nación. El proceso de convergencia de la contabilidad del sector público hacia estándares internacionales se dio a través de la Resolución 533 de 2015, por la cual se incorpora el marco normativo para entidades de gobierno y se fija la fecha para el periodo de aplicación obligatoria. Uno de los principales inconvenientes para la adopción del nuevo marco normativo es el período de transición, ya que las entidades públicas no cuentan con información financiera detallada; lo anterior, supone un problema relacionado con la cantidad de bienes que no tienen una cuantifica ción exacta. Varias entidades municipales del departamento del Quindío, realizaron este proceso; sin embargo, la información generada no es del todo clara, carece de una descripción detallada referente a los efectos ocasionados por la transición; en razón de lo anterior, se desconoce los cambios estructurales en las políticas contables y los impactos financieros en los procesos de reconocimiento, medición, declaración y presentación de los hechos económicos.

En busca de la solución a esta situación expuesta anteriormente, en este proyecto de investigación se realizó una descripción del impacto e incidencia en el estado de situación financiera de apertura, mediante la evaluación del cumplimiento de un cronograma de actividades; como también, el nivel de implementación de la Resolución 533 de 2015. Los resultados se pueden evidenciar a través de tablas de semaforización de impactos y gráficas de indicadores financieros; el estudio se realizó en la alcaldía del municipio de La Tebaida - Quindío, haciendo uso de herramientas de análisis basadas en el nuevo marco normativo. 


\section{Materiales y Métodos}

REVISTA INNOVA ITFIP, 7 (1). 89-109 $\quad$ DIC. 2020

La investigación desarrollada en la cual se fundamenta el presente artículo es cualitativa, descriptiva y documental, debido a que se inicia con una normativa general emitida por la Contaduría General de la Nación y se describe el impacto generado por la implementación de la Resolución 533 del 2015 en la alcaldía del municipio de Tebaida del departamento del Quindío El estudio presentó un carácter descriptivo porque se analizaron los resultados a través de la conceptualización teórica que lo fundamenta, teniendo en cuenta el manual de políticas contables y el estado de situación financiera de apertura a una entidad específica.

La información para el estudio fue obtenida mediante encuestas aplicadas a la entidad; la unidad de análisis del proyecto, es el Contador Público quien labora en el sitio objeto de estudio.

\section{Resultados y Discusión}

Con el propósito de determinar el nivel de implementación del marco normativo contenido en la Resolución 533 de 2015 al 31 de diciembre de 2017, se realizó un estudio a todo el proceso de migración de la contabilidad de la entidad, hacia normas internacionales; se llegó a un acuerdo de desarrollar el proceso en tres etapas como se observa en la tabla 1.

Tabla 1. Etapas del proceso de implementación de la Resolución 533 del 2015. Etapas del proceso de convergencia a normas internacionales de La administración municipal de La Tebaida, Quindío.

\begin{tabular}{|c|c|c|}
\hline Etapa & & Procedimientos \\
\hline 1 & $\begin{array}{c}\text { Diagnóstico y } \\
\text { dimensionamiento } \\
\text { de la entidad }\end{array}$ & $\begin{array}{l}\text { La finalidad fue identificar las diferencias entre los tratamientos contables } \\
\text { bajo la norma local y los requerimientos para la implementación de las } \\
\text { normas internacionales de la información financiera para el sector público, } \\
\text { conforme al nuevo marco aplicable para entidades de gobierno según la } \\
\text { Resolución } 533 \text { de } 2015\end{array}$ \\
\hline 2 & $\begin{array}{c}\text { Etapa de ejecución: } \\
\text { Capacitación y } \\
\text { políticas contables }\end{array}$ & $\begin{array}{l}\text { Para la vigencia que corresponde al periodo del } 2017 \text {, el comité técnico de } \\
\text { sostenibilidad contable de la administración municipal de la Tebaida, en } \\
\text { cumplimiento con las exigencias de la contaduría general de la nación, } \\
\text { realizó diversas capacitaciones y talleres al personal en las normas de } \\
\text { reconocimiento, medición, presentación y revelación de la información; de } \\
\text { igual forma, se realizó un diagnóstico al sistema de información para } \\
\text { establecer la adecuación del mismo a la nueva norma y se orientó para la } \\
\text { elaboración del manual de políticas contables bajo la nueva Resolución }\end{array}$ \\
\hline 3 & $\begin{array}{l}\text { Implementación del } \\
\text { nuevo marco } \\
\text { normativo y } \\
\text { elaboración del } \\
\text { estado de situación } \\
\text { financiera de } \\
\text { apertura }\end{array}$ & $\begin{array}{l}\text { La adecuada implementación de las políticas contables permitió mejorar el } \\
\text { desempeño organizacional y agilizar los procesos de toma de decisiones } \\
\text { financieras de la entidad; para lo cual, es necesario la comprensión e } \\
\text { interpretación que se debe tener al momento de elegir las normas que } \\
\text { integrarán el manual de políticas de la entidad. }\end{array}$ \\
\hline
\end{tabular}

Fuente: Elaboración propia a partir del instrumento de recolección de información. 
Por otra parte, el grupo de investigación "La función financiera para las organizaciones del departamento del Quindio", en cuanto al tema de convergencia dentro de sus objetivo está describir el impacto los procesos de adopción de la normatividad nacional e internacional de las entidades del sector público; para lo cual, desarrolló un instrumento de recolección de información que permitió realizar un descripción más detallada de algunos criterios o procesos de la entidad, herramienta que facilitó determinar el nivel de implementación e impacto de las actividades desarrolladas en la elaboración del estado de situación financiera de apertura -ESFA, bajo los lineamientos de la Resolución 533 de 2015, de los cuales se realiza una generalización a continuación

Desempeño de la gerencia en el proceso de transición hacia el nuevo marco normativo: El papel de la gerencia fue fundamental para llevar a cabo la migración de la contabilidad hacia Normas Internacionales, ella es la encargada de suministrar a la organización todos los recursos técnicos y humanos para la correcta aplicación del esquema de contabilidad del nuevo marco normativo. El gráfico 1. muestra porcentualmente el desempeño de las actividades ejecutadas con respecto a su implementación.

Gráfico $N^{\circ} 1$. Desempeño de la gerencia en la aplicación del nuevo marco normativo.

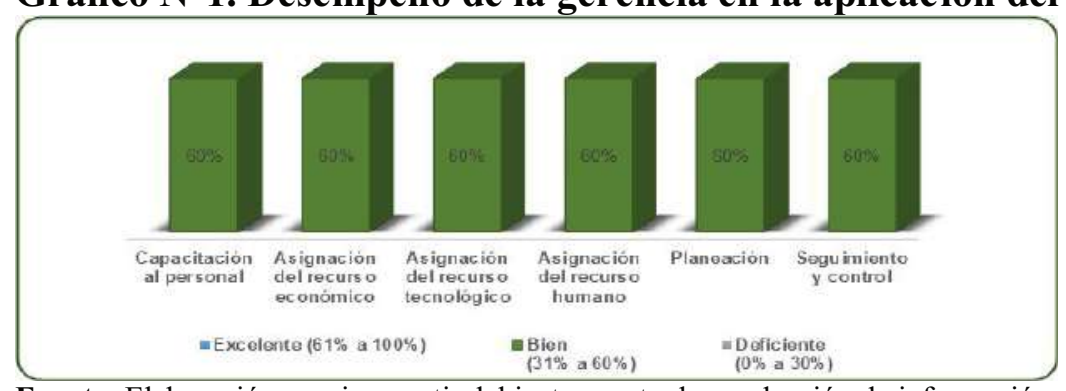

Fuente: Elaboración propia a partir del instrumento de recolección de información.

En términos generales el desempeño de la administración municipal en el proceso de transición hacia la nueva normatividad es aceptable, las actividades ejecutadas por la misma, se desarrollaron en su mayoría con un porcentaje admisible dentro del rango de evaluación.

Dificultades en el proceso de adopción de las normas internacionales de contabilidad pública: La transición a la norma requirió el apoyo permanente de las áreas gerenciales y contables, debido a que precisa cambios estructurales en la presentación de la información financiera y diversos aspectos adicionales a adoptar por parte del personal que consolida y prepara la información financiera.

De acuerdo con los resultados obtenidos a través de la aplicación del instrumento de recolección de información, se clasificaron las actividades realizadas en el proceso de adopción de la norma, según su grado de complejidad utilizando la escala de 1 al 9, siendo 9 el mayor grado de dificultad y 1 el menor, como se expresa en la tabla 2. 
REVISTA INNOVA ITFIP, 7 (1). 89-109 DIC. 2020

Tabla 2. Grado de dificultad en el proceso de adopción de la Resolución 533 del 2015.

\begin{tabular}{|l|c|}
\hline \multicolumn{1}{|c|}{ Actividades } & Dificultad \\
\hline Aplicación del instructivo 002 del 2015. & 3 \\
\hline Definición y elaboración del manual de políticas contables. & 5 \\
\hline Elaboración del estado de situación financiera de apertura & 8 \\
\hline Aplicación de matemáticas financieras. & 7 \\
\hline Adecuación del sistema de información contable. & 6 \\
\hline Preparación de las revelaciones, derivadas de la aplicación del nuevo marco normativo & 2 \\
\hline Medición y reconocimiento de activos y pasivos. & 7 \\
\hline Conciliación patrimonial. & 1 \\
\hline Apropiación del marco conceptual. & 3 \\
\hline
\end{tabular}

Fuente: Elaboración propia a partir del instrumento de recolección de información.

En la tabla $\mathrm{N}^{\circ} 2$, se evidencia un alto nivel de complejidad en las acciones, en razón a la aplicación de matemáticas financieras, medición y reconocimiento de activos y pasivos, la elaboración de los estados de situación financiera de apertura de la entidad, la adecuación del sistema de información contable y la medición y reconocimiento de los activos. Esto debido a todo el proceso que implicó el reconocimiento de las cuentas bajo el nuevo marco normativo, se recurrió a un ente externo para realizar los avalúos de los bienes muebles y propiedades, para posteriormente realizar el reconocimiento de cada cuenta en la nueva contabilidad.

Cumplimiento del cronograma de implementación: En cumplimiento con el periodo de preparación obligatoria y las actividades que se debían llevar a cabo según la Resolución 533 de 2015, la entidad elaboró un plan de acción para la adopción del nuevo marco normativo a 31 de diciembre de 2017, el cual fue presentado acorde a las fechas establecidas por la norma ante la contaduría general de la nación, organismos de inspección, vigilancia y control; por lo tanto, el cumplimiento de la entidad con respecto al desarrollo de estas actividades es del 100\%. Con respecto al periodo de aplicación según la Resolución 693 de 2016 la entidad cumplió el cronograma de actividades con sus respectivos soportes, anexos, evidencias, papeles de trabajo y los ajustes hacia la migración de saldos iniciales para la convergencia, además, realizó la actualización de los bienes inmuebles de la entidad, propiedad, planta y equipo según los avalúos realizados por el especialista contratado; de igual manera se realizaron los ajustes necesarios al manual de políticas el cual fue implementado como respaldo para la entidad en su nuevo marco contable.

Cumplimiento de la elaboración y presentación del plan de acción: La entidad cumplió con las actividades de su plan de acción como designación del responsable del proceso de convergencia, conformación del equipo de trabajo para la adopción del nuevo marco normativo, definición del cronograma de actividades para llevar a cabo el proceso de convergencia, estableció el plan de capacitación transversal que involucró las diferentes áreas de la entidad y por último se evaluó los impactos a nivel operativo, tecnológico, administrativo y financiero, dejando constancia de esto 
por escrito. Por otra parte, se analizó el nuevo marco técnico normativo para establecer las políticas contables aplicables a la entidad, modificando los procesos y procedimientos operativos y administrativos que fueron afectados con la determinación de las políticas contables, adecuando y fortaleciendo el recurso humano y tecnológico acorde a las nuevas necesidades.

Nivel de implementación del instructivo 002 de 2015 en la elaboración del estado de situación financiera de apertura: Considerando los resultados obtenidos a través del instrumento, se puede inferir que la misma preparó el estado de situación financiera de apertura-ESFA con base a los criterios establecidos en el instructivo 002 de 2015 y por lo tanto a los requerimientos de la Resolución 533 de 2015, dentro de los cuales se encuentra la presentación de los estados en las fechas establecidas, seguimiento de los procedimientos contables, guías de aplicación y referencia técnica de los criterios de reconocimiento, medición, revelación, presentación de los sucesos económicos.

Con la siguiente escala valorativa, se evaluó y presentó la semaforización del impacto en las actividades desarrolladas en el proceso de migración de la contabilidad.

\begin{tabular}{|c|l|}
\hline IMP ACTO ALTO (IA) & $\begin{array}{l}\text { Incidencias que conllevaron a un impacto de alta relevancia y } \\
\text { significancia. }\end{array}$ \\
\hline IMP ACTO MEDIO (IM) & $\begin{array}{l}\text { Incidencias que causaron a un impacto de moderada relevancia y } \\
\text { significancia. }\end{array}$ \\
\hline IMP ACTO BAJO (IB) & $\begin{array}{l}\text { incidencias que conllevaron a un impacto de baja relevancia e } \\
\text { importancia }\end{array}$ \\
\hline N/A & No aplica \\
\hline
\end{tabular}

La tabla 4, describe el nivel de implementación e impacto de las actividades desarrolladas por la entidad en el proceso de la elaboración del estado de situación financiera de apertura, de acuerdo con el instructivo 002 del 2015, para algunas cuentas de activo, pasivo y patrimonio a través de sus partidas, se midieron por el saldo a 31 de diciembre del 2017.

Tabla 4. Semaforización de impactos, cumplimiento de actividades e incidencia en las partidas del estado de situación financiera de apertura a 01 de enero de 2018.

\begin{tabular}{|c|c|c|c|c|c|c|}
\hline \multicolumn{7}{|c|}{ ACTIVO } \\
\hline \multirow[t]{2}{*}{ PARTIDA } & SI & $\mathrm{NO}$ & $\begin{array}{l}\mathrm{N} / \\
\mathrm{A}\end{array}$ & & & \\
\hline & 4 & 1 & 1 & $\begin{array}{c}\text { Análisis de la } \\
\text { materialidad de los } \\
\text { ajustes de las cuentas } \\
\text { para la determinación de } \\
\text { los saldos iniciales } \\
\text { IM }\end{array}$ & $\begin{array}{l}\text { Incidencia en la } \\
\text { complejidad y/o } \\
\text { esfuerzo en la } \\
\text { implementación } \\
\text { IA }\end{array}$ & $\begin{array}{c}\text { Incidencia en los } \\
\text { procesos, sistemas y } \\
\text { personal } \\
\text { IA }\end{array}$ \\
\hline $\begin{array}{c}\text { Inversiones de } \\
\text { administración de } \\
\text { liquidez }\end{array}$ & $\begin{array}{l}\text { las in } \\
\text { elabo } \\
\text { se cla } \\
\text { realiz } \\
2017 \\
\text { se mi }\end{array}$ & $\begin{array}{l}\text { iones } \\
\text { ón de } \\
\text { ó los } \\
\text { ajust } \\
\text { conc } \\
\text { on las }\end{array}$ & ot & $\begin{array}{l}\text { ministración de liquidez } \\
\text { o de situación financier } \\
\text { mentos de patrimonio q } \\
\text { able para eliminar el sal } \\
\text { valorizaciones asociad } \\
\text { siones por su saldo al } 3\end{array}$ & $\begin{array}{l}\text { e clasificaron al } \\
\text { de apertura al co } \\
\text { no tengan valor } \\
\text { registrado a } 31 \\
\text { con las inversio } \\
\text { de diciembre de }\end{array}$ & $\begin{array}{l}\text { nento de la } \\
\text { por lo tanto, } \\
\text { mercado, se } \\
\text { liciembre de } \\
\text { y por último } \\
\text {. }\end{array}$ \\
\hline
\end{tabular}


REVISTA INNOVA ITFIP, 7 (1). 89-109 DIC. 2020

\begin{tabular}{|c|c|c|c|c|c|c|}
\hline & \multicolumn{6}{|c|}{$\begin{array}{l}\text { La administración municipal de La Tebaida, cuenta con } 3 \text { socios los cuales son } \\
\text { Zona Franca, Serví Aseo y EPQ, lo que implicó analizar cada uno de los socios } \\
\text { para obtener la participación de estos, en las acciones frente a la administración } \\
\text { municipal, lo que conllevó a examinar si estos generaban rentabilidad, } \\
\text { beneficios, utilidades sociales, entre otras, se reflejó que EPQ no cumplía con su } \\
\text { participación, lo que llevó a realizar un análisis de medición posterior, para saber } \\
\text { si era una inversión u otra clase de activo y como resultado, se obtuvo que era } \\
\text { una subvención del gobierno. }\end{array}$} \\
\hline \multirow[t]{2}{*}{ PARTIDA } & SI & $\mathrm{NO}$ & $\begin{array}{c}\mathrm{N} / \\
\mathrm{A}\end{array}$ & & & \\
\hline & 3 & 1 & 0 & \begin{tabular}{|c} 
Análisis de la \\
materialidad de los \\
ajustes de las cuentas \\
para la determinación de \\
los saldos iniciales \\
IA
\end{tabular} & $\begin{array}{l}\text { Incidencia en la } \\
\text { complejidad y/o } \\
\text { esfuerzo en la } \\
\text { implementación } \\
\text { IA }\end{array}$ & $\begin{array}{c}\text { Incidencia en los } \\
\text { procesos, sistemas y } \\
\text { personal } \\
\text { IA }\end{array}$ \\
\hline \multirow{2}{*}{ Cuentas por cobrar } & \multicolumn{6}{|c|}{$\begin{array}{l}\text { Se realizó el ajuste contable para eliminar el saldo registrado a } 31 \text { de diciembre } \\
\text { de } 2017 \text { por concepto de provisiones asociadas a estas cuentas por cobrar, } \\
\text { afectando directamente el patrimonio en la cuenta impactos por transición al } \\
\text { nuevo marco de regulación, se evaluó si existe evidencia objetiva de deterioro de } \\
\text { las cuentas por cobrar y por último se acordó realizar el deterioro a } 31 \text { de } \\
\text { diciembre del } 2018 \text {. }\end{array}$} \\
\hline & \multicolumn{6}{|c|}{$\begin{array}{l}\text { La administración municipal recibe los impuestos directos que alimentan el } \\
\text { funcionamiento de la alcaldía, muchos de los predios que están al nombre del } \\
\text { municipio para que la cartera se redujera, la secretaria de planeación intervino; } \\
\text { además, se realizó una reclasificación de la cuenta de préstamos por cobrar, lo } \\
\text { que con llevó a que el rubro de ésta, pasara a cuentas por cobrar y quedará en } \\
\text { cero. }\end{array}$} \\
\hline \multirow[t]{2}{*}{ PARTIDA } & SI & $\mathrm{NO}$ & $\begin{array}{l}\mathrm{N} / \\
\mathrm{A}\end{array}$ & & & \\
\hline & 0 & 0 & 0 & \begin{tabular}{|c} 
Análisis de la \\
materialidad de los \\
ajustes de las cuentas \\
para la determinación de \\
los saldos iniciales \\
IA
\end{tabular} & $\begin{array}{l}\text { Incidencia en la } \\
\text { complejidad y/o } \\
\text { esfuerzo en la } \\
\text { implementación } \\
\text { IA }\end{array}$ & $\begin{array}{c}\text { Incidencia en los } \\
\text { procesos, sistemas y } \\
\text { personal } \\
\text { IA }\end{array}$ \\
\hline inventarios & \multicolumn{6}{|c|}{$\begin{array}{l}\text { Se realizó una depuración de los inventarios y hacer el avalúo de los bienes } \\
\text { inmuebles de la entidad, el cual se desarrolló a través del comité interno } \\
\text { interdisciplinario para poder analizar la información del valor de mercado con } \\
\text { variables del estado físico del elemento, lo cual permitió encontrar una manera } \\
\text { de valorar los bienes, para lo cual se requirió a un acto administrativo y se } \\
\text { estableció nuevamente su vida útil, a través de métodos de depreciación y nuevos } \\
\text { valores para el año } 2018 \text { como saldo inicial para bienes inmuebles. }\end{array}$} \\
\hline \multirow[t]{2}{*}{ PARTIDA } & SI & $\mathrm{NO}$ & $\begin{array}{l}\mathrm{N} / \\
\mathrm{A}\end{array}$ & & & \\
\hline & 9 & 2 & 0 & \begin{tabular}{|c|} 
Análisis de la \\
materialidad de los \\
ajustes de las cuentas \\
para la determinación de \\
los saldos iniciales \\
IA \\
\end{tabular} & $\begin{array}{l}\text { Incidencia en la } \\
\text { complejidad y/o } \\
\text { esfuerzo en la } \\
\text { implementación } \\
\text { IA }\end{array}$ & $\begin{array}{c}\text { Incidencia en los } \\
\text { procesos, sistemas y } \\
\text { personal } \\
\text { IA }\end{array}$ \\
\hline
\end{tabular}




\begin{tabular}{|c|c|c|c|c|c|c|}
\hline \multirow[t]{2}{*}{$\begin{array}{l}\text { Propiedades, } \\
\text { planta y equipo }\end{array}$} & \multicolumn{6}{|c|}{$\begin{array}{l}\text { Las propiedades, planta y equipo se midieron a través del comité técnico } \\
\text { interdisciplinario; para los bienes inmuebles por valor comercial el perito } \\
\text { determinó que era más los costos, por lo tanto, el valor se incrementó y para dar } \\
\text { su valor económico se avaluó a través del valor de la venta. } \\
\text { Se contrató un asesor externo para determinar los avalúos con efectos contables; } \\
\text { lo cual le permitió; a la entidad fijar la lista de predios pertenecientes a la } \\
\text { administración municipal de la Tebaida, se realizó un ajuste contable y se entró } \\
\text { en un proceso legal para verificar que el activo perteneciese a la entidad. }\end{array}$} \\
\hline & \multicolumn{6}{|c|}{$\begin{array}{l}\text { Se identificaron y clasificaron las propiedades, planta y equipo por su naturaleza, } \\
\text { como terrenos, maquinaria y equipo, plantas productoras, edificaciones o } \\
\text { muebles y enseres, entre otros. Se realizó el ajuste contable para eliminar las } \\
\text { provisiones de propiedades, planta y equipo reconocidas a } 31 \text { de diciembre de } \\
2017 \text {; de igual manera, se realizó el ajuste contable para eliminar las } \\
\text { valorizaciones de propiedades, planta y equipo reconocidas a } 31 \text { de diciembre de } \\
2017 \text { lo que conllevó a incrementar el mayor valor al activo }\end{array}$} \\
\hline \multirow[t]{2}{*}{ PARTIDA } & SI & $\mathrm{NO}$ & $\begin{array}{l}\mathrm{N} / \\
\mathrm{A}\end{array}$ & & & \\
\hline & 1 & 9 & 0 & $\begin{array}{c}\text { Análisis de la } \\
\text { materialidad de los } \\
\text { ajustes de las cuentas } \\
\text { para la determinación de } \\
\text { los saldos iniciales } \\
\text { IA }\end{array}$ & $\begin{array}{l}\text { Incidencia en la } \\
\text { complejidad y/o } \\
\text { esfuerzo en la } \\
\text { implementación } \\
\text { IA }\end{array}$ & $\begin{array}{l}\text { Incidencia en los } \\
\text { procesos, sistemas y } \\
\text { personal } \\
\text { IA }\end{array}$ \\
\hline $\begin{array}{l}\text { Bienes de uso } \\
\text { público }\end{array}$ & \multicolumn{6}{|c|}{$\begin{array}{l}\text { Los bienes de uso público, se identificaron y clasificaron por su naturaleza, } \\
\text { como infraestructura, parques, hemerotecas, bibliotecas o plazas, entre otros. } \\
\text { Además, estos se midieron con base en un avaluó externo y no se tuvo en } \\
\text { cuenta depreciaciones ni deterioros dado que su estimación tiende a } \\
\text { valorizarse. }\end{array}$} \\
\hline \multirow[t]{2}{*}{ PARTIDA } & SI & NO & $\begin{array}{l}\mathrm{N} \\
\mathrm{A}\end{array}$ & & & \\
\hline & 1 & 9 & 0 & $\begin{array}{c}\text { Análisis de la } \\
\text { materialidad de los } \\
\text { ajustes de las cuentas } \\
\text { para la determinación de } \\
\text { los saldos iniciales } \\
\text { IB }\end{array}$ & $\begin{array}{c}\text { Incidencia en la } \\
\text { complejidad y/o } \\
\text { esfuerzo en la } \\
\text { implementación } \\
\text { IB }\end{array}$ & $\begin{array}{l}\text { Incidencia en los } \\
\text { procesos, sistemas y } \\
\text { personal } \\
\text { IB }\end{array}$ \\
\hline $\begin{array}{l}\text { Bienes históricos y } \\
\text { culturales }\end{array}$ & \multicolumn{6}{|c|}{$\begin{array}{l}\text { Se realizó el reconocimiento a los bienes históricos y culturales y se procedió a } \\
\text { su medición al momento de la elaboración del estado de situación financiera de } \\
\text { apertura, para lo cual se identificaron los bienes que han sido declarados } \\
\text { mediante acto administrativo, se determinó los bienes no cumplen con los } \\
\text { criterios para ser clasificados como propiedades, planta y equipo, propiedades } \\
\text { de inversión o bienes de uso público, se identificaron los bienes históricos y } \\
\text { culturales por su naturaleza, como monumentos históricos, obras de arte o } \\
\text { lugares arqueológicos, entre otros, se midió cada partida del valor en libros al } \\
31 \text { de diciembre de } 2017 \text { y al valor de mercado o al costo de reposición } \\
\text { determinado a través de un avalúo técnico en la fecha de transición. }\end{array}$} \\
\hline \multirow[t]{2}{*}{ PARTIDA } & SI & $\mathrm{NO}$ & $\begin{array}{l}\mathrm{N} \\
\mathrm{A}\end{array}$ & & & \\
\hline & 2 & 3 & 1 & $\begin{array}{c}\text { Análisis de la } \\
\text { materialidad de los } \\
\text { ajustes de las cuentas }\end{array}$ & $\begin{array}{l}\text { Incidencia en la } \\
\text { complejidad y/o } \\
\text { esfuerzo en la } \\
\text { implementación }\end{array}$ & $\begin{array}{c}\text { Incidencia en los } \\
\text { procesos, sistemas y } \\
\text { personal } \\
\text { IB }\end{array}$ \\
\hline
\end{tabular}


REVISTA INNOVA ITFIP, 7 (1). 89-109 DIC. 2020

\begin{tabular}{|c|c|c|c|c|c|c|}
\hline & & & & $\begin{array}{c}\text { para la determinación de } \\
\text { los saldos iniciales } \\
\text { IB }\end{array}$ & IB & \\
\hline $\begin{array}{c}\text { Activos } \\
\text { intangibles }\end{array}$ & \multicolumn{6}{|c|}{$\begin{array}{l}\text { Se identificaron los activos intangibles, pero solo los adquiridos se midieron; } \\
\text { para luego realizar una transacción sin contraprestación, se tuvo en cuenta el } \\
\text { valor de mercado del activo en la fecha en que se adquirió más las mejoras, por } \\
\text { lo tanto se ajustaron los valores. }\end{array}$} \\
\hline \multirow[t]{2}{*}{ PARTIDA } & SI & NO & $\begin{array}{c}\mathrm{N} / \\
\mathrm{A}\end{array}$ & & & \\
\hline & 2 & 2 & 0 & \begin{tabular}{|c|} 
Análisis de la \\
materialidad de los \\
ajustes de las cuentas \\
para la determinación de \\
los saldos iniciales \\
IB \\
\end{tabular} & $\begin{array}{l}\text { Incidencia en la } \\
\text { complejidad y/o } \\
\text { esfuerzo en la } \\
\text { implementación } \\
\text { IB }\end{array}$ & $\begin{array}{c}\text { Incidencia en los } \\
\text { procesos, sistemas y } \\
\text { personal } \\
\text { IB }\end{array}$ \\
\hline $\begin{array}{l}\text { Deterioro del } \\
\text { valor de los } \\
\text { activos no } \\
\text { generados de } \\
\text { efectivo }\end{array}$ & \multicolumn{6}{|c|}{$\begin{array}{l}\text { Se definió una política contable para identificar los activos no generadores de } \\
\text { efectivo, dada su materialidad deban ser objeto de análisis de deterioro; } \\
\text { además, se esperó un año para medir los indicios de deterioro y por último, } \\
\text { pasado un año del periodo de aplicación, se calculó el valor de los indicios de } \\
\text { los activos recuperable. }\end{array}$} \\
\hline
\end{tabular}

\begin{tabular}{|c|c|c|c|c|c|c|}
\hline \multicolumn{7}{|c|}{ PASIVOS } \\
\hline \multirow[t]{2}{*}{ PARTIDA } & SI & $\mathrm{NO}$ & $\begin{array}{l}\mathrm{N} / \\
\mathrm{A}\end{array}$ & & & \\
\hline & 1 & 0 & 0 & $\begin{array}{c}\text { Análisis de la } \\
\text { materialidad de los } \\
\text { ajustes de las cuentas } \\
\text { para la determinación de } \\
\text { los saldos iniciales } \\
\text { IB } \\
\end{array}$ & $\begin{array}{c}\text { Incidencia en la } \\
\text { complejidad y/o } \\
\text { esfuerzo en la } \\
\text { implementación } \\
\text { IB }\end{array}$ & $\begin{array}{c}\text { Incidencia en los } \\
\text { procesos, sistemas y } \\
\text { personal } \\
\text { IB }\end{array}$ \\
\hline $\begin{array}{l}\text { Cuentas por } \\
\text { pagar }\end{array}$ & \multicolumn{6}{|c|}{$\begin{array}{l}\text { Las cuentas por pagar se reconocieron por su valor en libros a } 31 \text { de diciembre de } \\
2017 .\end{array}$} \\
\hline \multirow[t]{2}{*}{ PARTIDA } & SI & $\mathrm{NO}$ & $\begin{array}{l}\mathrm{N} / \\
\mathrm{A}\end{array}$ & & & \\
\hline & 8 & 2 & 0 & $\begin{array}{c}\text { Análisis de la } \\
\text { materialidad de los } \\
\text { ajustes de las cuentas } \\
\text { para la determinación de } \\
\text { los saldos iniciales } \\
\text { IA }\end{array}$ & $\begin{array}{l}\text { Incidencia en la } \\
\text { complejidad y/o } \\
\text { esfuerzo en la } \\
\text { implementación } \\
\text { IA }\end{array}$ & $\begin{array}{c}\text { Incidencia en los } \\
\text { procesos, sistemas y } \\
\text { personal } \\
\text { IA }\end{array}$ \\
\hline \multirow[t]{2}{*}{$\begin{array}{l}\text { Beneficios a los } \\
\text { empleados }\end{array}$} & \multicolumn{6}{|c|}{$\begin{array}{l}\text { La cuenta de beneficios a los empleados en la entidad se manejan a corto plazo y } \\
\text { post-empleo; para ello se identificó y clasificó las obligaciones presentes que la } \\
\text { entidad debe liquidar o pagar a los empleados dentro de los } 12 \text { meses siguientes a } \\
\text { la fecha de transición; de igual manera, se reconoció, como un pasivo, las } \\
\text { obligaciones a corto plazo que no hayan sido reconocidas a } 31 \text { de diciembre de } \\
\text { 2017, por el valor de la obligación derivada de los beneficios definidos a la fecha } \\
\text { de transición, después de deducir cualquier pago anticipado y se realizó el ajuste } \\
\text { contable para eliminar los pasivos a corto plazo a } 31 \text { de diciembre de } 2017 \text {. }\end{array}$} \\
\hline & \multicolumn{6}{|c|}{$\begin{array}{l}\text { Se replanteó la estructura de reclasificación, lo cual resultó complejo para el } \\
\text { procedimiento }\end{array}$} \\
\hline
\end{tabular}




\begin{tabular}{|c|c|c|c|c|c|c|}
\hline PARTIDA & SI & $\mathrm{NO}$ & $\begin{array}{l}\mathrm{N} / \\
\mathrm{A}\end{array}$ & & & \\
\hline & 7 & 2 & 0 & \begin{tabular}{|} 
Análisis de la \\
materialidad de los \\
ajustes de las cuentas \\
para la determinación de \\
los saldos iniciales \\
IA
\end{tabular} & $\begin{array}{l}\text { Incidencia en la } \\
\text { complejidad y/o } \\
\text { esfuerzo en la } \\
\text { implementación } \\
\text { IA }\end{array}$ & $\begin{array}{l}\text { Incidencia en los } \\
\text { procesos, sistemas y } \\
\text { personal } \\
\text { IA }\end{array}$ \\
\hline \multirow[t]{3}{*}{ Provisiones } & \multicolumn{6}{|c|}{$\begin{array}{l}\text { Se identificaron y clasificaron las obligaciones probables, legales y/o implícitas, } \\
\text { que posea la entidad y sobre las cuales tenga incertidumbre en relación a su } \\
\text { cuantía y/o vencimiento. Se identificaron las obligaciones no reconocidas a } 31 \text { de } \\
\text { diciembre de } 2017 \text { y se midieron por la mejor estimación del desembolso que se } \\
\text { requeriría, para cancelar la obligación presente o transferir a un tercero en la } \\
\text { fecha de transición, sé identificó si la entidad ha asumido compromisos para } \\
\text { llevar a cabo planes de reestructuración, sé midió la provisión por } \\
\text { reestructuración por la mejor estimación del desembolso que se requeriría para } \\
\text { cancelar la obligación presente o para transferirla a un tercero en la fecha de } \\
\text { transición. }\end{array}$} \\
\hline & \multicolumn{6}{|c|}{$\begin{array}{l}\text { Se identificó si la entidad posee contratos de carácter oneroso, que pudieran } \\
\text { afectar directamente el patrimonio en la cuenta impactos por transición al Nuevo } \\
\text { Marco de Regulación. }\end{array}$} \\
\hline & \multicolumn{6}{|c|}{$\begin{array}{l}\text { Se aplicó la Resolución } 353 \text { de la agencia nacional de defensa jurídica que habla } \\
\text { sobre la provisión contable de los pasivos contingentes por pagar en sentencias, } \\
\text { litigios y demandas en contra. Se estableció una provisión legal para pérdidas y } \\
\text { compra de activos. Para la administración municipal la más relevante es la cuenta } \\
\text { de sentencias, lo cual se aprende en el proceso a no materializarla. }\end{array}$} \\
\hline
\end{tabular}

\begin{tabular}{|c|c|c|c|c|c|c|}
\hline \multicolumn{7}{|c|}{ PATRIMONIO } \\
\hline \multirow[t]{2}{*}{ PARTIDA } & SI & NO & $\begin{array}{c}\mathrm{N} / \\
\mathrm{A}\end{array}$ & & & \\
\hline & 7 & 2 & 0 & \begin{tabular}{|c|} 
Análisis de la \\
materialidad de los \\
ajustes de las cuentas \\
para la determinación de \\
los saldos iniciales \\
IA
\end{tabular} & $\begin{array}{l}\text { Incidencia en la } \\
\text { complejidad y/o } \\
\text { esfuerzo en la } \\
\text { implementación } \\
\text { IA }\end{array}$ & $\begin{array}{l}\text { Incidencia en los } \\
\text { procesos, sistemas y } \\
\text { personal } \\
\text { IA }\end{array}$ \\
\hline $\begin{array}{l}\text { Capital fiscal, } \\
\text { resultado de } \\
\text { ejercicios } \\
\text { anteriores. }\end{array}$ & $\begin{array}{l}\text { La ad } \\
\text { valor } \\
\text { prepa } \\
\text { norm } \\
\text { actua } \\
\text { reduj } \\
\text { entid } \\
\text { evide } \\
\text { algun }\end{array}$ & $\begin{array}{l}\text { istrac } \\
\text { de I } \\
\text { con } \\
\text { par } \\
\text { ione } \\
\text { table } \\
\text { on ba } \\
\text { un a }\end{array}$ & & $\begin{array}{l}\text { unicipal realizó el recon } \\
\text { bre del } 2017 \text {; además la } \\
\text { men de contabilidad pú } \\
\text { ades de gobierno, aume } \\
\text { s valores de las cuentas } \\
\text { en la depuración de los } \\
\text { as cuentas por cobrar a } \\
\text { o en el valor de las prov } \\
\text { ivamente explotados y }\end{array}$ & $\begin{array}{l}\text { imiento de las cl } \\
\text { iferencia entre el } \\
\text { ica anterior y el } \\
\text { ó considerablem } \\
\text { propiedades, pl } \\
\text { rechos que se su } \\
\text { contribuyentes; } \\
\text { iones y el recono } \\
\text { los cuales se rec }\end{array}$ & $\begin{array}{l}\text { as por su } \\
\text { rimonio, } \\
\text { nuevo marco } \\
\text { con base a las } \\
\text { y equipo y se } \\
\text { ía tenía la } \\
\text { otra parte, se } \\
\text { iento de } \\
\text { un beneficio. }\end{array}$ \\
\hline
\end{tabular}

\begin{tabular}{|c|c|c|c|c|c|c|}
\hline \multicolumn{7}{|c|}{ OTROS TEMAS } \\
\hline PARTIDA & SI & NO & $\begin{array}{c}\mathrm{N} / \\
\mathrm{A}\end{array}$ & & & \\
\hline & 4 & 0 & 0 & $\begin{array}{c}\text { Análisis de la } \\
\text { materialidad de los } \\
\text { ajustes de las cuentas } \\
\text { para la determinación de } \\
\text { los saldos iniciales }\end{array}$ & $\begin{array}{l}\text { Incidencia en la } \\
\text { complejidad y/o } \\
\text { esfuerzo en la } \\
\text { implementación } \\
\text { IA }\end{array}$ & $\begin{array}{l}\text { Incidencia en los } \\
\text { procesos, sistemas y } \\
\text { personal } \\
\text { IA }\end{array}$ \\
\hline
\end{tabular}


REVISTA INNOVA ITFIP, 7 (1). 89-109 DIC. 2020

\begin{tabular}{|c|c|c|c|c|c|c|}
\hline & & & & IA & & \\
\hline $\begin{array}{c}\text { Efecto del } \\
\text { saneamiento } \\
\text { contable, efecto } \\
\text { por la aplicación } \\
\text { del régimen de } \\
\text { contabilidad } \\
\text { pública }\end{array}$ & \multicolumn{6}{|c|}{$\begin{array}{l}\text { Se identificó si la entidad tiene saldos y se reclasificaron los saldo a la cuenta } \\
\text { impactos por transición al nuevo marco de regulación; además, se permitió reflejar } \\
\text { el patrimonio, por lo tanto se aplicó la ley } 1819 \text { del } 2019 \text { la cual dio la posibilidad } \\
\text { de hacer mejoras al saneamiento a través de la ley } 717 \text { del } 2001 \text {, la ley } 1739 \text { del } \\
2014 \text { y la ley } 1753 \text { del } 2015 \text {. }\end{array}$} \\
\hline \multirow[t]{2}{*}{ PARTIDA } & SI & $\mathrm{NO}$ & $\begin{array}{l}\mathrm{N} / \\
\mathrm{A}\end{array}$ & & & \\
\hline & 2 & 0 & 0 & \begin{tabular}{|c|} 
Análisis de la \\
materialidad de los \\
ajustes de las cuentas \\
para la determinación de \\
los saldos iniciales \\
IA \\
\end{tabular} & $\begin{array}{l}\text { Incidencia en la } \\
\text { complejidad y/o } \\
\text { esfuerzo en la } \\
\text { implementación } \\
\text { IA }\end{array}$ & $\begin{array}{l}\text { Incidencia en los } \\
\text { procesos, sistemas y } \\
\text { personal } \\
\text { IA }\end{array}$ \\
\hline $\begin{array}{l}\text { Activos } \\
\text { contingentes }\end{array}$ & \multicolumn{6}{|c|}{$\begin{array}{l}\text { Se identificó si la entidad posee activos y se determinó la naturaleza de estos y se } \\
\text { incluyeron dentro de las cuentas de orden deudoras que se hayan podido medir. }\end{array}$} \\
\hline \multirow[t]{2}{*}{ PARTIDA } & SI & NO & $\begin{array}{l}\mathrm{N} / \\
\mathrm{A}\end{array}$ & & & \\
\hline & 2 & 0 & 0 & \begin{tabular}{|c|} 
Análisis de la \\
materialidad de los \\
ajustes de las cuentas \\
para la determinación de \\
los saldos iniciales \\
IA \\
\end{tabular} & $\begin{array}{l}\text { Incidencia en la } \\
\text { complejidad y/o } \\
\text { esfuerzo en la } \\
\text { implementación } \\
\text { IA }\end{array}$ & $\begin{array}{l}\text { Incidencia en los } \\
\text { procesos, sistemas y } \\
\text { personal } \\
\text { IA }\end{array}$ \\
\hline $\begin{array}{l}\text { Pasivos } \\
\text { contingentes }\end{array}$ & \multicolumn{6}{|c|}{$\begin{array}{l}\text { Se identificó si la entidad posee pasivos y se determinó la naturaleza de estos y se } \\
\text { incluyeron dentro de las cuentas de orden acreedoras los cuales se hayan podido } \\
\text { medir; además, se valoraron los pasivos por litigios y demandas que se iban a } \\
\text { perder y se evaluó el riesgo. }\end{array}$} \\
\hline
\end{tabular}

Con el propósito de describir el impacto administrativo y financiero generado tras la implementación de la Resolución 533 de 2015, se aplicaron herramientas de análisis, con las cuales fue posible determinar el porcentaje que tuvo cada partida dentro del estado de situación financiera de apertura; así mismo, identificar cada una de sus variaciones con referencia al estado de situación financiera bajo norma local.

Se dispuso de la herramienta financiera de análisis horizontal, donde se tuvo en cuenta la variación absoluta y relativa, lo cual permitió establecer el incremento o disminución de una cuenta en un periodo de tiempo determinado; análisis que es importante para identifi car si la entidad tiene una distribución equitativa de sus activos y de acuerdo a las necesidades competitivas, financieras y operativas, como se muestra en la tabla 5, donde se realizó análisis a los estados financieros del municipio de La Tebaida. 
Tabla 5. Estado de situación financiera comparativos con análisis horizontal.

\begin{tabular}{|c|c|c|c|c|c|}
\hline & \multirow[t]{2}{*}{ CUENTA } & \multirow{2}{*}{$\begin{array}{c}\text { BALANCE RCP } \\
\begin{array}{l}\text { Al } 31 \text { de diciembre } \\
\text { del } 2017\end{array}\end{array}$} & \multirow{2}{*}{$\begin{array}{c}\text { ESFA } \\
\text { Al } 01 \text { de enero del } \\
2018\end{array}$} & \multicolumn{2}{|c|}{ ANALISIS HORIZONTAL } \\
\hline & & & & $\begin{array}{l}\text { VARIACION } \\
\text { ABSOLUTA }\end{array}$ & $\begin{array}{l}\text { VARIACION } \\
\text { RELATTIVA }\end{array}$ \\
\hline 1. & ACTIVO & $\$ 49.619 .576 .690$ & $\$ 50.622 .458 .835$ & $\$ 1.002 .882 .145$ & $2,02 \%$ \\
\hline \multicolumn{2}{|c|}{ ACTIVO CORRIENTE } & $\$ 28.386 .351 .266$ & $\$ 28.859 .945 .043$ & $\$ 473.593 .777$ & $1,67 \%$ \\
\hline 1.1 & $\begin{array}{l}\text { EFECTIVO Y } \\
\text { EQUIVALENTES AL } \\
\text { EFECTIVO } \\
\end{array}$ & $\$ 12.728 .176 .098$ & $\$ 12.728 .176 .098$ & $\$ 0$ & $0,00 \%$ \\
\hline 1.2 & $\begin{array}{l}\text { INVERSIONES E } \\
\text { INSTRUMENTOS } \\
\text { DERIVADOS }\end{array}$ & $\$ 442.915 .200$ & $\$ 879.485 .746$ & $\$ 436.570 .546$ & $98,57 \%$ \\
\hline 1.3 & CUENTAS POR COBRAR & $\$ 11.133 .369 .666$ & $\$ 15.252 .283 .199$ & $\$ 4.118 .913 .533$ & $37,00 \%$ \\
\hline 1.4 & PRÉST AMOS POR COBRAR & $\$ 4.081 .890 .302$ & $\$ 0$ & $-\$ 4.081 .890 .302$ & $-100,00 \%$ \\
\hline \multicolumn{2}{|c|}{ ACTIVO NO CORRIENTE } & $\$ 21.233 .225 .424$ & $\$ 21.762 .513 .793$ & $\$ 529.288 .368$ & $2,49 \%$ \\
\hline 1.5 & INVENT ARIOS & $\$ 970.224$ & $\$ 0$ & - \$970.224 & $-100,00 \%$ \\
\hline 1.6 & $\begin{array}{l}\text { PROPIEDADES, PLANTA Y } \\
\text { EQUIPO }\end{array}$ & $\$ 7.622 .258 .756$ & $\$ 8.989 .760 .295$ & $\$ 1.367 .501 .538$ & $17,94 \%$ \\
\hline 1.7 & $\begin{array}{l}\text { BIENES DE BENEFICIO Y } \\
\text { USO PÚBLICO E } \\
\text { HISTÓRICOS Y } \\
\text { CULTURALES } \\
\end{array}$ & $\$ 0$ & $\$ 85.888 .268$ & $\$ 85.888 .268$ & $100,00 \%$ \\
\hline 1.9 & OTROS ACTIVOS & $\$ 13.609 .996 .444$ & $\$ 12.686 .865 .230$ & $-\$ 923.131 .214$ & $-6,78 \%$ \\
\hline 2. & PASIVOS & $\$ 2.532 .447 .613$ & $\$ 2.532 .447 .613$ & \$0 & $0,00 \%$ \\
\hline \multicolumn{2}{|c|}{ PASIVO CORRIENTE } & $\$ 2.214 .428 .368$ & $\$ 2.270 .513 .955$ & $\mathbf{\$ 5 6 . 0 8 5 . 5 8 7}$ & $2,53 \%$ \\
\hline 2.2 & $\begin{array}{l}\text { EMISIÓN Y COLOCACIÓN } \\
\text { DE TÍTULOS DE DEUDA }\end{array}$ & $\$ 1.227 .188 .628$ & $\$ 0$ & $-\$ 1.227 .188 .628$ & $-100,00 \%$ \\
\hline 2.3 & PRÉSTAMOS POR PAGAR & $\$ 0$ & $\$ 1.249 .538 .419$ & $\$ 1.249 .538 .419$ & $100,00 \%$ \\
\hline 2.4 & CUENTAS POR PAGAR & $\$ 491.849 .106$ & $\$ 525.584 .902$ & $\$ 33.735 .796$ & $6,86 \%$ \\
\hline 2.5 & $\begin{array}{l}\text { BENEFICIOS A LOS } \\
\text { EMPLEADOS }\end{array}$ & $\$ 495.390 .634$ & $\$ 495.390 .634$ & $\$ 0$ & $0,00 \%$ \\
\hline \multicolumn{2}{|c|}{ PASIVO NO CORRIENTE } & $\$ 318.019 .245$ & $\$ 261.933 .658$ & -\$56.085.587 & $-17,64 \%$ \\
\hline 2.7 & PROVISIONES & $\$ 261.933 .658$ & $\$ 261.933 .658$ & $\$ 0$ & $0,00 \%$ \\
\hline 2.9 & OTROS PASIVOS & $\$ 56.085 .587$ & $\$ 0$ & $-\$ 56.085 .587$ & $-100,00 \%$ \\
\hline 3 & PATRIMONIO & $\$ 47.087 .129 .077$ & $\$ 48.090 .011 .223$ & $\$ 1.002 .882 .146$ & $2,13 \%$ \\
\hline \multirow[t]{2}{*}{3.1} & HACIENDA PÚBLICA & \$47.087.129.077 & $\$ 48.090 .011 .223$ & $\$ 1.002 .882 .146$ & $2,13 \%$ \\
\hline & PASIVO + PATRIMONIO & $\$ 49.619 .576 .690$ & $\$ 50.622 .458 .835$ & & \\
\hline
\end{tabular}

Fuente: Elaboración propia a partir de los estados financieros 2017 de la Alcaldía municipal de la Tebaida Quindío. (Quindio, 2018)

Una vez aplicado el análisis horizontal, al estado de situación financiera de apertura en comparación con el balance bajo norma local, se evidenció algunos impactos que sufrió la estructura de la contabilidad de la entidad, principalmente por el incremento considerable del patrimonio, ya que éste se vio afectado por el ejercicio contable del saneamiento para la transición al nuevo marco normativo a causa de la aplicación de los lineamientos de este. En la Tabla 6 se presenta el análisis horizontal de forma detallada. 
REVISTA INNOVA ITFIP, 7 (1). 89-109 DIC. 2020

Tabla 6. Análisis horizontal del estado de situación financiera de apertura (ESFA)

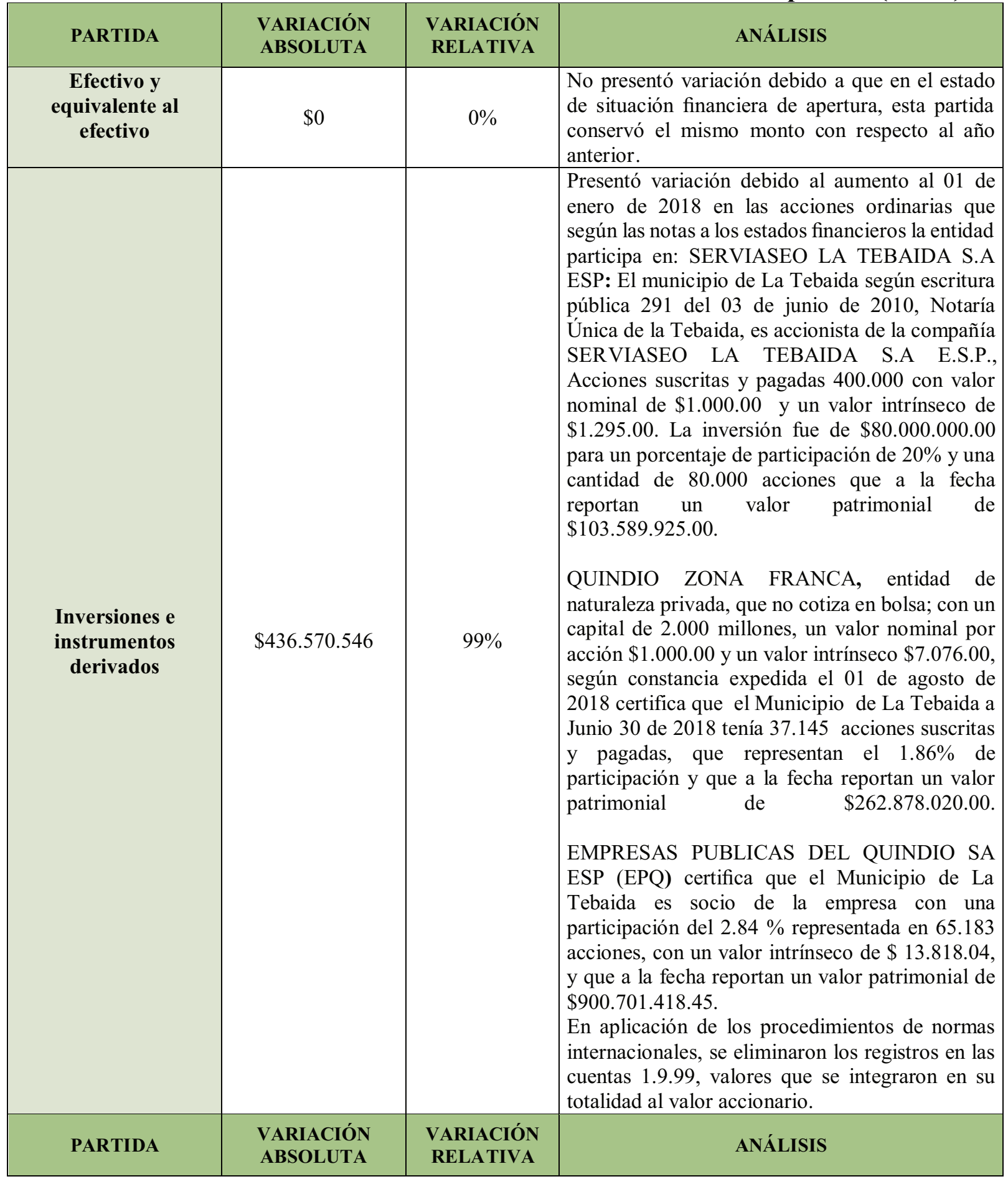




\begin{tabular}{|c|c|c|c|}
\hline Cuentas por cobrar & $\$ 4.118 .913 .533$ & $37 \%$ & $\begin{array}{l}\text { Presentó un aumento debido a la reclasificación } \\
\text { de las cuentas tras la aplicación del instructivo } 002 \\
\text { de } 2015 \text {. }\end{array}$ \\
\hline $\begin{array}{l}\text { Prestamos por } \\
\text { cobrar }\end{array}$ & $-\$ 4.081 .890 .302$ & $-100 \%$ & $\begin{array}{l}\text { Presentó disminución debido a que bajo el nuevo } \\
\text { marco normativo ésta cuenta es reemplazada por } \\
\text { la cuenta "cuentas por cobrar", el saldo que venía } \\
\text { al } 31 \text { de diciembre de } 2017 \text { fue trasladado al } \\
\text { estado de situación financiera de apertura del } 01 \\
\text { de enero de } 2018 \text { como una Cuenta por Cobrar. }\end{array}$ \\
\hline Inventario & $-\$ 970.224$ & $-100 \%$ & $\begin{array}{l}\text { Presentó una disminución debido al ajuste } \\
\text { contable, afectando directamente al patrimonio en } \\
\text { la cuenta de impactos por transición al nuevo } \\
\text { marco de regulación tras la aplicación del } \\
\text { instructivo } 002 \text { de } 2015 \text {. }\end{array}$ \\
\hline $\begin{array}{c}\text { Propiedades, planta } \\
\text { y equipo }\end{array}$ & $\$ 1.367 .501 .538$ & $17,94 \%$ & $\begin{array}{l}\text { Presentó un aumento debido a que según las notas } \\
\text { a los estados financieros nos dice que "de acuerdo } \\
\text { a la aplicación de los procesos de actualización } \\
\text { en la implementación de las normas } \\
\text { internacionales de contabilidad para el sector } \\
\text { público, las Propiedades planta y equipo han sido } \\
\text { reconocidos con un valor de } \$ 47.601 .520 .934 .11 \text {. } \\
\text { A Diciembre } 31 \text { de } 2017 \text { la Propiedad Planta y } \\
\text { Equipos se contabilizaban por valor de } \\
\$ 7.622 .258 .756 .11 \text {, sufriendo un incremento en el } \\
\text { estado de situación financiera de apertura a un } \\
\text { valor de } \$ 8.989 .760 .294 .56 \text { y al } 31 \text { de diciembre } \\
\text { de } 2018 \text { a } \$ 47.601 .520 .934 .11 \text {, con afectación a la } \\
\text { cuenta de patrimonio" }\end{array}$ \\
\hline $\begin{array}{l}\text { Bienes de beneficio y } \\
\text { uso público e } \\
\text { históricos y } \\
\text { culturales }\end{array}$ & $\$ 85.888 .268$ & $100 \%$ & $\begin{array}{l}\text { Presentaron un aumento esto debido a la } \\
\text { reclasificación de las cuentas según la aplicación } \\
\text { del instructivo } 002 \text { del } 2015\end{array}$ \\
\hline PARTIDA & $\begin{array}{l}\text { VARIACIÓN } \\
\text { ABSOLUTA }\end{array}$ & $\begin{array}{l}\text { VARIACIÓN } \\
\text { RELATIVA }\end{array}$ & ANÁLISIS \\
\hline Otros activos & $-\$ 923.131 .214$ & $-7 \%$ & $\begin{array}{l}\text { Presentó una disminución debido a la } \\
\text { reclasificación de las cuentas de bienes de } \\
\text { beneficio y uso público e histórico y cultural; } \\
\text { además de la cuenta de valorizaciones la cual } \\
\text { afectó directamente al patrimonio. }\end{array}$ \\
\hline Pasivo & $\$ 0$ & $0 \%$ & $\begin{array}{l}\text { No presentó variación debido a que en el estado } \\
\text { de situación financiera de apertura esta partida } \\
\text { conservó el mismo monto con respecto al año } \\
\text { anterior. }\end{array}$ \\
\hline Hacienda pública & $\$ 1.002 .882 .146$ & $2 \%$ & $\begin{array}{l}\text { Presentó aumento debido al saneamiento al } 31 \text { de } \\
\text { diciembre del } 2017 \text { de las cuentas de la partida de } \\
\text { patrimonio y el impacto por la transición al nuevo } \\
\text { marco normativo al } 01 \text { de enero del } 2018 \text {. }\end{array}$ \\
\hline
\end{tabular}

Fuente: Elaboración propia a partir de los estados financieros 2017 de la alcaldía municipal de la Tebaida Quindío y notas a los estados financieros al 31 de diciembre del 2017. (Quindio, 2018) 
Con el propósito de identificar el impacto financiero generado por el proceso de transición al nuevo marco normativo, se aplicaron herramientas de análisis que permiten ponderar y evaluar los resultados revelados por los estados financieros evaluados, estas herramientas se conocen como indicadores financieros; las cuales permiten diagnosticar la capacidad de endeudamiento, generar liquidez y utilidades; de igual manera, facilitan el análisis de la situación financiera de la entidad de forma individual, con lo cual se pueden realizar comparaciones con las mismas, con otras entidades o dependencias para identificar las variaciones que presentó de un periodo a otro, tras la implementación de la Resolución 533 de 2015.

La materialidad de los impactos, se midió a través de una escala de semaforización de impactos generados tras la implementación de la Resolución 533 de 2015 como se describe a continuación, a través del desarrollo de los indicadores financieros que se realizaron de acuerdo a las cifras obtenidas del análisis comparativo.

\begin{tabular}{|c|l|c|}
\hline $\begin{array}{c}\text { NIVEL DE } \\
\text { IMPACTO }\end{array}$ & \multicolumn{1}{|c|}{ DESCRIPCIóN } & COLOR \\
\hline ALTO & $\begin{array}{l}\text { La implementación de la Resolución tuvo un impacto significativo en la preparación } \\
\text { del estado de situación financiera de apertura. }\end{array}$ & $\begin{array}{l}\text { La implementación de la Resolución tuvo un impacto moderado en la preparación del } \\
\text { estado de situación financiera de apertura. }\end{array}$ \\
\hline MEDIO & $\begin{array}{l}\text { La implementación de la Resolución no tuvo un impacto significativo en la } \\
\text { preparación del estado de situación financiera de apertura. }\end{array}$ \\
\hline BAJO & \\
\hline
\end{tabular}
Fuente: Elaboración propia.

Los indicadores financieros se calcularon teniendo en el concepto y su fórmula razón corriente, prueba acida y capital neto trabajado.

Datos obtenidos del análisis horizontal o comparativo. En la tabla 7, se resumen los resultados del indicar razón corriente, en la tabla 8, los de la prueba acida y en la tabla 9, el de capital neto trabajado.

Tabla 7. Razón corriente

\begin{tabular}{|r|r|r|r|}
\hline \multicolumn{2}{|c|}{ Enero 1 - 2018 } & \multicolumn{2}{c|}{ Diciembre 31-2017 } \\
\hline$\$ 27.980 .459 .299$ & 12,32 & $\$ 28.386 .351 .266$ & 12,82 \\
\hline$\$ 2.270 .513 .955$ & & $\$ 2.214 .428 .368$ & \\
\hline
\end{tabular}

Fuente: Elaboración propia

El resultado de la tabla 7, indica la capacidad de cubrir la entidad sus obligaciones a corto plazo, lo cual, disminuyeron en un $11 \%$

Tabla 8. Prueba acida

\begin{tabular}{|r|c|r|r|}
\hline Enero 1-2018 & \multicolumn{2}{c|}{ Diciembre 31-2017 } \\
\hline$\$ 27.980 .459 .299$ & 12,32 & $\$ 28.385 .381 .042$ & 12,82 \\
\hline$\$ 2.270 .513 .955$ & & $\$ 2.214 .428 .368$ & \\
\hline \multicolumn{4}{|c|}{ Fuente: Elaboración propia }
\end{tabular}


El resultado de la tabla 8, evidencia que el municipio tiene completa capacidad de atender sus obligaciones, sin necesidad de contar con la venta de sus inventarios, teniendo en cuenta que las organizaciones gubernamentales no manejan grandes volúmenes de los mismos.

Tabla 9. Capital neto de trabajo

Fuente: Elaboración propia

\begin{tabular}{|l|c|}
\hline Enero 1 - 2018 & Diciembre 31-2017 \\
\hline$\$ 25.709 .945 .344$ & $\$ 26.171 .922 .898$ \\
\hline
\end{tabular}

En análisis al capital neto de trabajo tabla 9, se puede evidenciar que para el año 2017, después de cancelar el total de las obligaciones corrientes, la entidad cuenta con \$26.171.922.898 para atender las obligaciones que surjan en el curso normal de las operaciones, mientras que para el año 2018, una vez la entidad canceló el total de sus obligaciones corrientes, le quedaron \$25.709.945.344 para atender las obligaciones que surjan en el curso normal de las operaciones, lo que indica que tras la implementación del nuevo marco normativo disminuyó su liquidez en \$461.977.544.

En la tabla 10, se analiza a través de la escala de semaforización el impacto de implementación de la Resolución.

Tabla 10. Indicadores de liquidez.

\begin{tabular}{|c|c|c|c|c|}
\hline \multicolumn{5}{|c|}{ Impacto de implementación de la Resolución } \\
\hline \multirow{2}{*}{$R C=\frac{\text { Activo corriente }}{\text { Pasivo corriente }}$} & $\begin{array}{l}\text { Diciembre } 31- \\
2017\end{array}$ & Enero 1 - 2018 & variación & Impacto \\
\hline & 12,82 & 12,32 & $-0,11$ & \\
\hline \multicolumn{5}{|c|}{ PRUEBA DE EFECTIVO } \\
\hline \multirow{2}{*}{$P A=\frac{\text { Activo corriente }- \text { Inventario }}{\text { Pasivo corriente }}$} & $\begin{array}{c}\text { Diciembre 31- } \\
2017\end{array}$ & Enero 1-2018 & variación & Impacto \\
\hline & 12,82 & 12,32 & $-0,11$ & \\
\hline \multicolumn{5}{|c|}{ CAPITAL NETO DE TRABAJO } \\
\hline \multirow[t]{2}{*}{ CTN $=$ Activo corriente - Pasivo corriente } & $\begin{array}{c}\text { Diciembre 31- } \\
2017\end{array}$ & Enero 1 - 2018 & variación & Impacto \\
\hline & $\$ 26.171 .922 .898$ & $\$ 25.709 .945 .344$ & -461.977 .544 & \\
\hline
\end{tabular}

Fuente: Elaboración propia

Posteriormente al análisis de los resultados obtenidos tras la aplicación de los indicadores de liquidez, se evidencia que la entidad cuenta con una liquidez de 12.82 y 12.71 para el periodo comparativo, lo que significa que por cada peso de la obligación, se contaba con estos indicadores para respaldar la deuda, teniendo en cuenta que la variación no es mayor a 1 que es lo ideal, por lo tanto, se entiende que está en un promedio de baja liquidez, considerando una disminución de 0.11 para el estado de situación financiera de apertura.

Por otra parte, se pudo evidenciar que la entidad no depende en absoluto de los inventarios ya que para el 2017 y 1 de Enero de 2018, cuentan con 12.82 y 12.71 respectivamente para atender todas sus obligaciones corrientes, con una variación negativa 0,11 . 


\section{Conclusiones}

El municipio de La Tebaida, como entidad fundamental de la división políticoadministrativa del Estado, le corresponde prestar los servicios públicos que determine la ley, construir las obras que demande el progreso local, ordenar el desarrollo de su territorio, promover la participación comunitaria, el mejoramiento social y cultural de sus habitantes y cumplir las demás funciones que le asignen la constitución y las leyes.

El desempeño de la administración municipal en el proceso de transición hacia el nuevo marco normativo cumplió con los estándares propuestos por la contaduría general de la nación, esto debido que la entidad ejecutó las actividades relacionadas con el proceso de convergencia. Se puede evidenciar que todas las actividades tuvieron respaldo por parte de los directivos de la entidad, con el apoyo del comité de sostenibilidad contable de la administración municipal que asumieron la responsabilidad de la planeación, seguimiento y control de este proceso.

Se presentó un alto nivel de complejidad en acciones como la aplicación de matemáticas financieras, medición y reconocimiento de activos y pasivos, la elaboración del estado de situación financiera de apertura de la entidad; la adecuación del sistema de información contable y la medición y reconocimiento de los activos, lo anterior debido a que todo el proceso implicó el reconocimiento de las cuentas bajo el nuevo marco normativo.

La entidad implementó el nuevo marco normativo en su totalidad para dar cumplimiento a la implementación de la norma, para lo cual parametrizó las cuentas contables en el sistema, lo que permitió registrar la contabilidad bajo la nueva norma.

Se evidenció que las políticas contables fueron adecuadas para cada uno de los elementos del estado de situación financiera, exceptuando de algunas partidas en las cuales no se especificó el método de medición, el tiempo de vida útil y los criterios para el reconocimiento.

El análisis e interpretación de los estados financieros, requirió de herramientas específicas de valoración, con las cuales se obtuvo cifras porcentuales de participación de cada una de las cuentas con sus respectivas variaciones; las más significativas, fueron el Activo en cuanto a efectivo y equivalente al efectivo, inversiones e instrumentos derivados y el Pasivo en las provisiones, partidas hicieron parte del saneamiento contable para la transición al nuevo marco normativo, lo cual tuvo un impacto significativo en el patrimonio de la entidad. 
La administración municipal debe seguir cumpliendo efectivamente con todos los requisitos expuestos por la Contaduría General de la Nación para ejecutar eficientemente las Normas Internacionales de Contabilidad del Sector Público; además establecer todas aquellas acciones y estrategias necesarias para que exista un adecuado y oportuno flujo de información y documentación hacia el área contable, con el propósito de garantizar el reconocimiento y revelación de todos los hechos económicos de la misma.

\section{Referencias bibliográficas}

Alcaldía de La Tebaida Q. (2018). Manual de políticas contables. La Tebaida Q.

Alcaldía municipal la Tebaida Q. (s.f.). Alcaldía de la Tebaida Q. Obtenido de http:/www.latebaida-quindio.gov.co/

Antunez, C. (2009). Crecimiento Económico (Modelo de crecimiento económico). Obtenido de: https://www.academia.edu/31924381/ECONOM\%C3\%8DA_CRECIMIENTO_ECON\%C 3\%93MICO.

Arango, D., \& Torres, A. G. (2014). Análisis del proceso de armonización de las normas internacionales de contabilidad e información financiera. Revista contexto, 3, 132-145.

Cardona, J. V . (2019). Programa de Gobierno. La Tebaida Quindío.

CGN. (2010). Armonización a estándares internacionales de contabilidad pública, la experiencia de la contaduría general de la nación. Textos de contabilidad pública, 2,7-85.

CGN. (2010). Normatividad de la entidad. Bogotá: Contaduría general de la nación.

CGN. (08 de Octubre de 2015). Contaduría General de la Nación. Obtenido de http://www.contaduria.gov.co/documents/20127/36444/Res \%2B533.pdf/b513cc87-772604ab-02e4-8691544220c6?t=1558381851097.

CGN. (08 de Octubre de 2015). Contaduría General de la Nación. Obtenido de http://www.contaduria.gov.co/documents/20127/38135/Instructivo+No.+002+de+2015.pdf/ $\underline{\text { lb42b6e1-2ffb-aa54-b6b4-654ce1497d9b?t=1577373296905. }}$.

CGN. (2015). Marco conceptual para la preparación y presentación de la información financiera de entidades de gobierno. Bogotá: Contaduría general de la nación. 
REVISTA INNOVA ITFIP, 7 (1). 89-109 DIC. 2020

CGN. (2016). La contabilidad y su utilidad en el sector público. Bogotá: Textos de contabilidad pública.

Contaduría General de la Nación (CGN). (01 de octubre de 2012). Contaduría General de la Nación. Obtenido de http://www.contaduria.gov.co/cartas-circulares

IFAC. (2019). Ifac.org.

INCP. (2015). Análisis financiero mediante indicadores para la toma de decisiones. Obtenido de https://www.incp.org.co/analisis-financiero-mediante-indicadores-para-la-toma-dedecisiones/.

IPSASB. (2019). Ipsasb.org. Obtenido de https://www.ipsasb.org.

Méndez, C.E. (2010). Metodología diseño y desarrollo del proceso de investigación con énfasis en ciencias empresariales, $4^{\mathrm{a}}$. Ed, Limusa. México.

Obando Cárdenas, J., Pulgarín Giraldo, H., \& Franco Ruiz, R. (2015). La contabilidad y su utilidad en el sector público. Bogotá: Contaduría General de la Nación.

Obando, J. A. (3 de Julio de 2017). Beneficios tributarios como consecuencia de los aportes a la responsabilidad social empresarial del subsector salud del Municipio de Santiago de Cali. Obtenido de http://revistas.ugca.edu.co/index.php/contexto/article/view/649/1012

Parra, N. d., \& Forero, D. L. (03 de Julio de 2017). Los beneficios que genera ser una empresa socialmente responsable en Colombia. Obtenido de Universidad de la Salle: https://revistas.lasalle.edu.co/index.php/ls/article/view/4029.

Quindío, C. G. (01 de enero de 2018). Contraloría General del Quindío.

República, C. d. (20 de Julio de 1991). Secretaria del Senado. Obtenido de http://www.secretariasenado.gov.co/senado/basedoc/constitucion_politica_1991.html.

República, C. d. (26 de enero de 1993). Secretaria del Senado. Obtenido de http://www.secretariasenado.gov.co/senado/basedoc/ley_0042_1993.html

República, C. d. (23 de Julio de 1996). Secretaria del Senado. Obtenido de http://www.secretariasenado.gov.co/senado/basedoc/ley 0298 1996.html

República, C. d. (13 de Julio de 2009). Secretaria del Senado. Obtenido de http://www.secretariasenado.gov.co/senado/basedoc/ley 1314 2009.html. 
Tebaida, A. d. (2011). Alcaldía Municipal la Tebaida (Q).

Urbano, J. (2014). La influencia de las NIIF del sector privado en las NICSP desde la perspectiva del interés público en Colombia. Bogotá.

Verdugo, J. C. (2016). El Papel De Las Normas Internacionales de Contabilidad del sector público. Caso México. Santander: Tesis. 